\title{
Von kritisch zu post-kritisch (?), Dominanz in der Generalisierung, Datenlücken in Citizen Science, „Giant Snakes" als Amazonas, und Konstruktion(en) von Portolankarten sowie einer Modellbahnkartographie
}

\author{
Dennis Edler ${ }^{1}$
}

Accepted: 31 August 2021 / Published online: 17 September 2021

(c) The Author(s) 2021

Liebe Leserinnen und Leser,

die Relevanz von sowohl Themenvielfalt als auch -spezialisierungen stand bereits im Fokus des Editorials zum vorausgehenden Heft dieses Jahrgangs. Bevor die Dezemberausgabe neueste Entwicklungen zur raumbezogenen Visualisierung mit Methoden und Techniken virtueller und erweiterter Realitäten thematisiert, bietet Ihnen dieses Varia-Heft - der Titel verrät es bereits - Einblicke in aktuelle Forschungsarbeiten zu vielseitigen Themen:

Die Reihe der sechs wissenschaftlichen Fachartikel beginnt inhaltlich durchaus polarisierend: Olaf Kühne (Tübingen) rekapituliert die Entwicklung der Kritischen Kartographie seit den 1980er Jahren, einschl. einer Kritik an der Kritischen Kartographie und einem theoriegeleiteten Vorschlag einer „Post-kritischen Kartographie “. Dabei repräsentiert das Präfix „Post “ nicht allein die zeitliche Dimension (im Sinne eines ,nach “), sondern bezieht sich ebenfalls auf die inhaltliche Entkoppelung gesetzter Schwerpunktperspektiven der Kritischen Kartographie.

Ein Beitrag zur kartographischen Generalisierung stammt von Mathias Gröbe und Dirk Burghardt (Dresden). Die Autoren stellen Methoden zur Auswahl von Punktmerkmalen vor, die für einen konkreten Maßstab gelten und auf numerischen Attributen basieren. Zudem wird ein neuer Ansatz beschrieben, der auf Dominanz beruht und - als der minimale Abstand zum nächstgelegenen Punkt mit einem höheren Wert - räumliche Positionen mit Attributwerten verbindet. Die beschriebenen Methoden werden als Plug-in „Point selection algorithms “ für QGIS bereitgestellt.

Dennis Edler

Dennis.Edler@ruhr-uni-bochum.de

1 Ruhr University Bochum, Bochum, Germany
Das Autorenteam Tom Hoyer, Julia Moritz und Jana Moser (Leipzig) adressiert die aktuelle Problematik, das Geodatensätze, die im Kontext von Citizen Science-Initiativen erfasst werden, durch ihre Lückenhaftigkeit der Kartographie besondere Herausforderungen bescheren. Durch das Fallbeispiel des Projekts „Artenvielfalt erleben “ wird vorgestellt, wie entsprechende Datenlücken in (Web-)Karten implementiert werden können, um die Aussagekraft der Daten hervorzuheben. Mit einer empirischen Erhebung werden zusätzliche Interpretationshilfen evaluiert.

Terry Bahill und Gary Gitzen (Tucson, AZ, USA) thematisieren ein Phänomen in historischen Karten des 16. Jahrhunderts, in denen der Amazonas von der Atlantikküste bis zu den Anden wiederholt als gigantische Schlange repräsentiert wurde. In einer vergleichenden Analyse wurden unter Anwendung eines mathematischen Modells verschiedene historische Karten hinsichtlich der kartographisch repräsentierten Form des Amazonas untersucht, um neue Erkenntnisse zur Evolution und zu den Zusammenhängen der markanten schlangenartigen Darstellungsweise abzuleiten.

Peter Mesenburg (Essen) widmet sich ebenfalls einem Thema der Historischen Kartographie. Er weist durch ein Beispiel der manuellen Kartierung nach, dass Portolankarten, die u.a. für ihre sehr hohe Genauigkeit bekannt sind, mit der Kreisschnittmethode, eines der ältesten Kartierverfahren, konstruiert werden konnten.

Ein hochschulübergreifendes Autorenteam aus der Geographie um Olaf Kühne (Tübingen) befasst sich mit kartographischen Darstellungen in der Fachliteratur zu Modelleisenbahnen. Dabei werden dominierende Kategorien vorgestellt und der vielseitige Konstruktionsprozess, einschl. der sozialen Konstruktion von (idealisierten) Landschaften, diskutiert. 


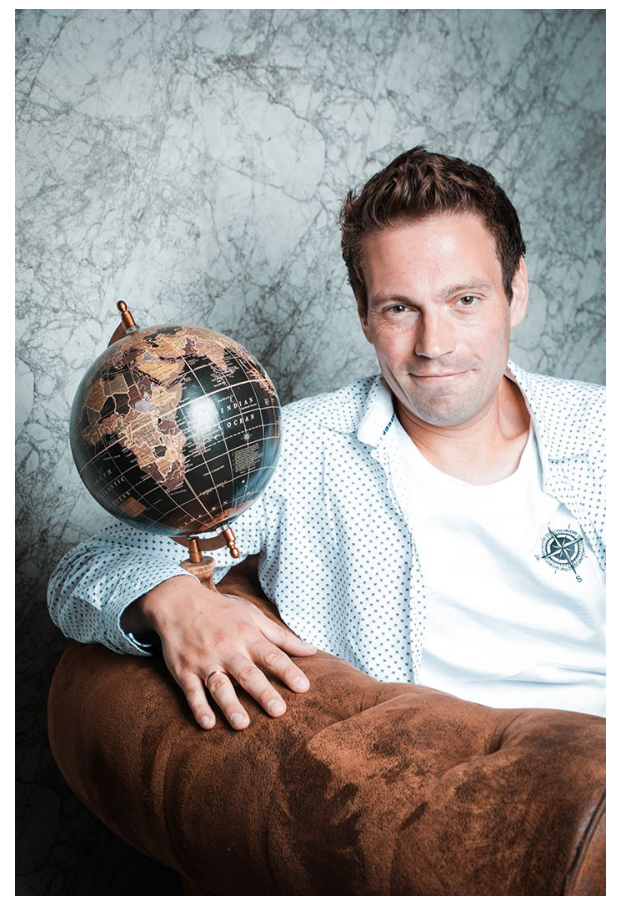

Viel Freude beim Lesen, ggf. auch beim Verfassen von Leserbriefen (?!) - und herzliche Grüße

Dennis Edler

Funding Open Access funding enabled and organized by Projekt DEAL.

Open Access This article is licensed under a Creative Commons Attribution 4.0 International License, which permits use, sharing, adaptation, distribution and reproduction in any medium or format, as long as you give appropriate credit to the original author(s) and the source, provide a link to the Creative Commons licence, and indicate if changes were made. The images or other third party material in this article are included in the article's Creative Commons licence, unless indicated otherwise in a credit line to the material. If material is not included in the article's Creative Commons licence and your intended use is not permitted by statutory regulation or exceeds the permitted use, you will need to obtain permission directly from the copyright holder. To view a copy of this licence, visit http://creativecommons.org/licenses/by/4.0/. 